\title{
Network Modeling and Performance Analysis under Time-varying Channel and General Arrival of M2M Services
}

\author{
$\mathrm{Fu} \mathrm{Li}$ \\ Department of Communication Engineering \\ Jilin University \\ ChangChun, China \\ 971740586@qq.com
}

\begin{abstract}
To investigate and forecast the traffic characteristics of important M2M service--Small data service, we modeled its arrival process and analyzed the effects it has on current wireless network. By simulating service logic and designing traffic generation function of typical M2M small data service on network simulation platform (OPNET), we modeled packet inter-arrival time of M2M aggregate traffic with general distribution $\mathrm{Gm}$. Considering the time-varying feature of wireless channel, MMPP-2 is used to model the service process of WLAN access network. Based on the queuing theory, we solve $G m / M M P P / 1 / K$ model under general arrival of M2M small data service and time-varying channel, then we obtained the performance metrics of the queuing system. The results indicate that (1) Hurst parameter of M2M aggregate traffic is approximately estimated as 0.7 , (2) M2M small data service has the characteristics of delay-tolerance, as a result, system blocking rate can be effectively reduced by increasing the buffer size in the error-prone wireless environment, this solution is feasible.
\end{abstract}

Key words-M2M, small data service, traffic model, time-varying wireless channel.

\section{INTRODUCTION}

M2M service refers to all the services related to machine communications. The number of M2M devices is becoming trillion in the near future. Considering the characteristics of WLAN, such as high transmission rate, simple network topology and easy to deploy, it should act as the bearing network of M2M applications. Using the simulation platform, we analyze the flow characteristics of M2M services based on the behavior features of M2M devices and then establish the arrival model of M2M aggregated traffic. Queuing theory is used to investigate the system performance of M2M bearing network under time-varying channel. Moreover, we explore the method to reduce the blocking rate.

Most of the researches on traffic modeling based on queuing theory use mathematical models to describe the arrival process of services for the sake of reducing the difficulties in solving queuing models ${ }^{[1][2][3]}$. In the aspect of the solution of the $G / M / 1 / K$ queuing model, Sunggon Kim derived the stationary distribution of the queue length of $G / M / 1 / K$ model with two-stage service policy ${ }^{[4]}$. Some researches investigate relationship between $M / G / 1 / K$ and $G / M / 1 / K$ queuing systems ${ }^{[5]}$. To enhance the system performance, Jau-Chuan Ke considered the single vacation model $G / M / 1 / K$ with $\mathrm{N}$ threshold policy ${ }^{[6]}$; Mohsin Iftikhar solved the multi-class $G / M / 1$ queuing system with self-similar input and non-preemptive priority ${ }^{[3]}$. However,

\author{
Xue-fen Chi ,Jia-sheng Zhang, Xu-dong Li \\ Department of Communication Engineering \\ Jilin University \\ ChangChun, China \\ chixf@jlu.edu.cn \\ zhangjs52061730@126.com
}

all above researches about $G / M / 1 / K$ queuing model focus on the theoretical analysis and mathematical solutions and there are few people paying close attention to the general arrival distribution of specific services in real networks; On the other hand, at present, the negative exponential distribution is widely used to model the network service time, but the time-varying characteristics of wireless channel makes it impossible to model the service process of wireless network used to bear MTC services accurately.

In our research, the packet generation behavior of three kinds of M2M small data services is simulated on the simulation platform (OPNET), and the packet inter-arrival time distribution $G m$ and Hurst parameter $H$ of M2M aggregated traffic are obtained. Taking the time-varying characteristics of wireless channel into account, we model the network service time with MMPP-2, and an exact analysis of $G m / M M P P / 1 / K$ model is carried out. The system performance metrics such as system blocking probability and average sojourn time are all derived. According to the delay-tolerance characteristics of M2M small data service, the method to reduce the packet blocking rate is to increase the buffer size in error-prone wireless environment. The results obtained in this paper are helpful for wireless resource management in order to provide QoS guarantee for large-scale application like M2M services.

\section{SIMULATION AND MODELING OF M2M SERVICES}

Typical M2M small data services in WLAN are simulated using OPNET. Based on the analysis of the flow characteristics of M2M small data services, we design the service logic and derive the general arrival distribution- $G m$ of M2M aggregated traffic by processing the real-time traffic data collected on the network simulation platform.

\section{A. Simulation of M2M Services}

The M2M services can be classified into 7 categories, containing 35 types. Among these, Auto Meter Reading (S1), Fleet Management (S2) and Automatic Vending Machine (S3) are analyzed in this paper.

The number of M2M devices is set according to WLAN capacity. ON-OFF traffic source is used to generate M2M traffic. We design the service logic of these three kinds of services and set the parameters according to the behavioral characteristics of M2M small data services. Packet inter-arrival time and the number of arrival packets in unit time are captured respectively, which help derive the 
general arrival distribution- $G m$ of M2M aggregated traffic. The network topology is shown in Figure 1.

S1 service has the characteristic of low mobility, and each S1 terminal transmits packets periodically. The behavior of a single S2 terminal is regular, while the aggregation behavior is random; For S3 service, packets are transmitted through event trigger and terminals are rarely moved. All of these services have small packet size. According to service logic, service behavior and network capacity, the number of devices is set 100 in each subnet, the parameters of different kinds of the M2M devices are the same as [10].

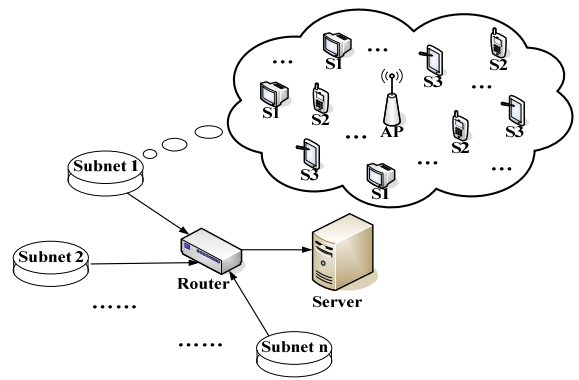

Figure 1 Communication scenario of M2M services in WLAN

We estimate the Hurst Parameter using R/S Method, shown in Table 1.

TABle 1 The HuRst Parameter of M2M Services

\begin{tabular}{|c|c|c|c|c|}
\hline Service Type & S1 & S2 & S3 & $\begin{array}{c}\text { Aggregation } \\
\text { Service }\end{array}$ \\
\hline Hurst Parameter & 0.5027 & 0.6290 & 0.6039 & 0.7012 \\
\hline
\end{tabular}

Observing Table 1, we find that the three kinds of M2M small data services have weak self-similarity, which results from the constant packet sizes of M2M services and the low randomness of the packet generation of devices. However, the self-similarity of aggregated traffic is strong.

\section{B. Probability Distribution of Inter-arrival Time of M2M Services}

To investigate the traffic characteristics of M2M small data services in specific network environment, general arrival distribution of M2M services is established using the data captured on the simulation platform ${ }^{[13]}$. We use $0.001 \mathrm{~s}$ as a time slot, and then the inter-arrival time is quantified. The probability distribution $G m$ of packet inter-arrival time of M2M services is derived by processing a large amount of inter-arrival time slots. The probability distribution of packet inter-arrival time whose number of time slots is between 0 and 500 is shown in Figure 2.

As is known to all, in the real network exists the aggregated traffic of all kinds of services, therefore, the arrival of M2M aggregated traffic is used as the input of the queuing model.

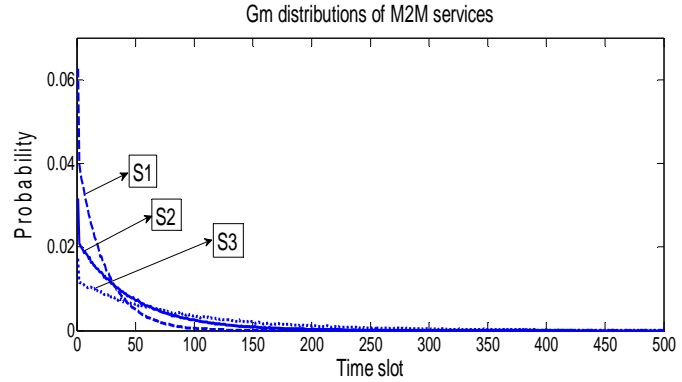

(a) $G m$ distribution of three M2M services

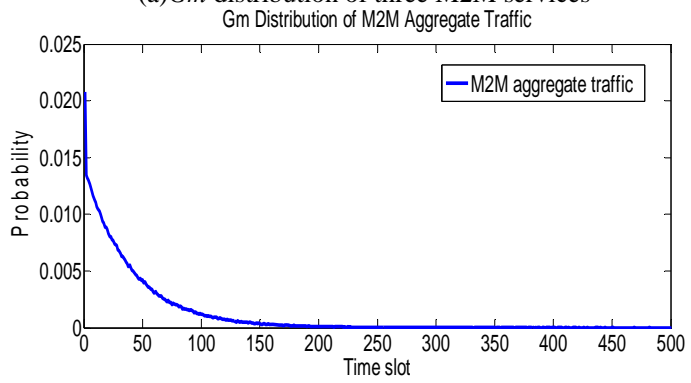

(b) $G m$ distribution of M2M aggregate traffic Figure $2 \mathrm{Gm}$ distribution of M2M services

\section{THE SOLUTION AND ANALYSIS OF $G m / M M P P / 1 / K$ MODEL}

Wireless channel is time-varying because of the affection of noise and interference. In WLAN, when the number of M2M terminals that requesting access, the average data rate of each work station changes accordingly. Therefore, negative exponential distribution is not able to describe the service time distribution of wireless network. In this case, wireless channel state is specified with two phases-'good' and 'bad', and the average service rate is different in different phases. Then MMPP model is used to characterize the service process of WLAN, shown as Figure 3.

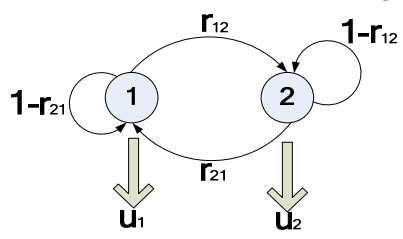

Figure 3 MMPP model

In Figure 3, phase 1 and phase 2 represent good channel and bad channel respectively and $r_{12}$ is the transfer probability from phase 1 to phase 2 while $r_{21}$ from phase 2 to phase 1 . The average service rate of phase 1 is $\mu_{l}$ and that of phase 2 is $\mu_{2}, \mu_{1}>\mu_{2}$

The infinitesimal generation matrix $\mathbf{R}$ and Poisson rate matrix $\Lambda$ are as follows.

$$
\mathbf{R}=\left[\begin{array}{cc}
-r_{12} & r_{12} \\
r_{21} & -r_{21}
\end{array}\right], \quad \boldsymbol{\Lambda}=\left[\begin{array}{cc}
u_{1} & 0 \\
0 & u_{2}
\end{array}\right]
$$

We get the stationary probability of each phase of MMPP model by computing $\theta_{1}$ and $\theta_{2} . \theta_{1}$ is the stationary probability of phase 1 and $\theta_{2}$ is that of phase 2 .

$$
\theta_{1}=r_{21} /\left(r_{12}+r_{21}\right)
$$




$$
\theta_{2}=r_{12} /\left(r_{12}+r_{21}\right)
$$

The average service rate of the whole system is $\mu$, it follows that

$$
u=\left(u_{1} \cdot r_{21}+u_{2} \cdot r_{12}\right) /\left(r_{12}+r_{21}\right)
$$

The MMPP phase at the time slot $t$ is denoted by $J(t), t \geqslant$ 0 , which is irreducible and ergotic. The number of packets served successfully during an interval of length $t$ is presented by $A(t)$. Then the probability that $n$ packets are successfully served during a service time interval and MMPP reaches phase $j$ at the end of the service time, starting from phase $i$ can be derived.

$$
\begin{gathered}
p_{i j}(n, t)=P\{A(t)=n, J(t)=j \mid A(0)=0, J(0)=i\} \\
i, j \in(1,2)
\end{gathered}
$$

The transmission probability of instantaneous service rate of MMPP model is given by

$$
\mathbf{P}(t)=e^{\mathbf{R} t}=\mathbf{I}-\frac{-1}{r_{12}+r_{21}}\left(e^{-\left(r_{12}+r_{21}\right) t}-1\right) \mathbf{R}
$$

Then $\mathrm{m} \times \mathrm{m}$ matrix $\mathbf{P}(n, t)$ can be carried out.

$$
\mathbf{P}(n, t)=\left\{p_{i j}(n, t)\right\}=\operatorname{diag}\left(\theta_{1}, \theta_{2}\right) \mathbf{P}(\boldsymbol{t}) \operatorname{diag}\left(\frac{\left(u_{1} \cdot t\right)^{n}}{n !} e^{-u_{1} \cdot t}, \frac{\left(u_{2} \cdot t\right)^{n}}{n !} e^{-u_{2} \cdot t}\right)
$$

The $(i, j)$ th element in $\mathbf{P}(n, t)$ denotes the conditional probability of reaching the phase $j$ and having $n$ packets served during an interval of length $t$, given that we start with phase $i$ at $t=0$.

$X(t)$ denotes the number of packets in the buffer queue at time slot $t$, and $t_{n}$ denotes the moment that the $n$th packet arrives. Then $X_{n}=X\left(t_{n}{ }^{+}\right)$and $J_{n}=J\left(t_{n}{ }^{+}\right)$are the state of the queuing model and the phase of MMPP after $t_{n}$ respectively. Therefore, a two-dimensional discrete time embedded Markov chain can be given by $\left\{X_{n}, J_{n}, n \geqslant 0\right\}$. The probability that $n$ packets are served during the time interval between two neighboring arrival packets is denoted.

$$
S_{n}=\sum_{t=0}^{\infty} \mathbf{P}(n, t) P_{m}(t)
$$

Then, we compute the transition probability matrix $\mathbf{P}$ as

$$
\mathbf{P}=\left[\begin{array}{cccccc}
0 & 1 & & & & \\
0 & 1-S_{0} & S_{0} & & & \\
0 & 1-\sum_{i=0}^{1} S_{i} & S_{1} & S_{0} & & \\
0 & 1-\sum_{i=0}^{2} S_{i} & S_{2} & S_{1} & \ddots & \\
\vdots & \vdots & \vdots & \vdots & \ddots & \\
0 & 1-\sum_{i=0}^{k-1} S_{i} & S_{k-1} & S_{k-2} & \cdots & S_{1}+S_{0}
\end{array}\right]
$$

Let $\pi_{n}$ be the stationary probability of $X n, \pi=\left(\pi_{0}, \pi_{1}, \ldots\right.$, $\left.\pi_{K}\right)$. By solving the equations $\boldsymbol{\pi} \mathbf{P}=\boldsymbol{\pi}$ and $\boldsymbol{\pi} \mathbf{e}=1$, we can get the stationary probability of each queue state, where $\mathbf{e}=(1,1, \ldots, 1)^{\mathrm{T}}$ is a column vector of which every element is 1 . Then the average queue length $\mathrm{Q}$, the blocking rate $\mathrm{L}$ and the average sojourn time $\mathrm{W}$ can be computed as follows:

$$
\begin{aligned}
& \mathrm{Q}=\sum_{k=0}^{K} \pi_{k} \cdot k \\
& \mathrm{~L}=\pi_{K} \cdot 100 \% \\
& \mathrm{~W}=\mu \cdot \mathrm{Q}
\end{aligned}
$$

Firstly, we investigate the QoS of M2M aggregated traffic in changeable wireless network environment. By controlling the phase transition probability of MMPP model, we simulate the time-varying characteristics of wireless channel. We set the service rate of phase $1 \mu_{1}=0.06$, and that of phase $2 \mu_{2}=0.03$ and define 5 channel state levels representing different quality levels of channel (quality decreases with channel state levels' growth). The stationary probability of different phases of MMPP is set in Table 2.

Set the buffer size $K=10,15,20$ (packets), the blocking rate $\mathrm{L}$ and the average sojourn time $\mathrm{W}$ are derived under different channel state levels.

TABLE 2 STATE LEVEL AND CORRESPONDING PHASE STABLE PROBABILITY

\begin{tabular}{ccc} 
& OF MMPP \\
\hline State Level & Probability in Phase 1 & Probability in Phase 2 \\
\hline 1 & 0.7 & 0.3 \\
2 & 0.6 & 0.4 \\
3 & 0.5 & 0.5 \\
4 & 0.4 & 0.6 \\
5 & 0.3 & 0.7 \\
\hline
\end{tabular}

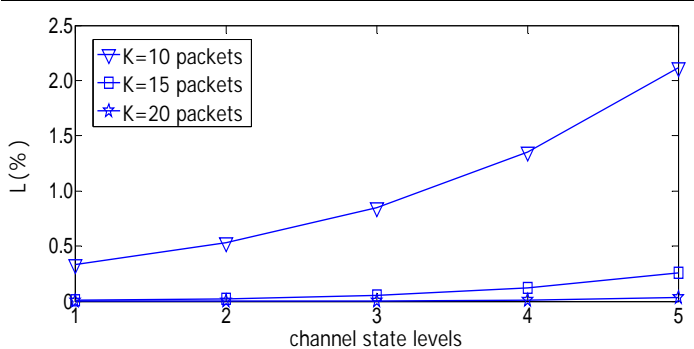

Figure 4 System blocking probability in different channel state levels

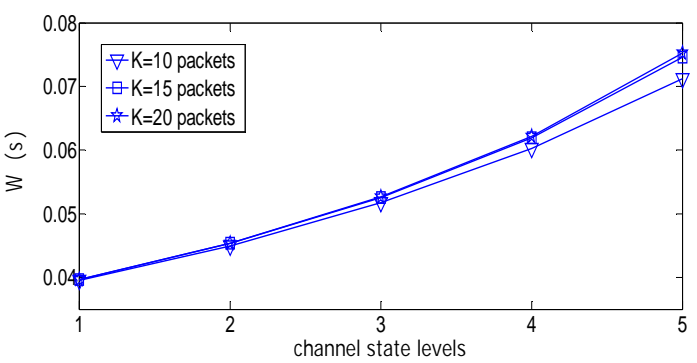

Figure 5 Average sojourn time under different channel state levels

As Figure 4 and Figure 5 show, when the quality of wireless channel becomes poor, the $\mathrm{L}$ and $\mathrm{W}$ both increase and the system performance decreases. Furthermore, we find that the performance deterioration trend slows down with the buffer size increasing. Accordingly, taking the delay-tolerance characteristics of M2M small data services into account, we explore the impact that the buffer size makes on the $\mathrm{L}$.

In real networks, most systems require $\mathrm{L}<0.1 \%$. In the queuing model $G m / M M P P / 1 / K$, we set $r_{12}=0, \quad r_{21}=1$, representing the best channel quality. To insure $\mathrm{L}=0.1 \%$, we compute the buffer size $\mathrm{K}$ and obtain that $\mathrm{K}$ should equal to 
10 approximately. Considering wireless channel is time-varying, then we investigate how to adjust the buffer size to guarantee the system blocking rate requirement when the channel quality decreases. According to the five groups of parameters in Table 3, we carry out the exact analysis of the $G m / M M P P / 1 / K$ queuing model respectively and derive the $\mathrm{L}$ and $\mathrm{W}$ as buffer size changes, shown as Figure 6 and Figure 7.

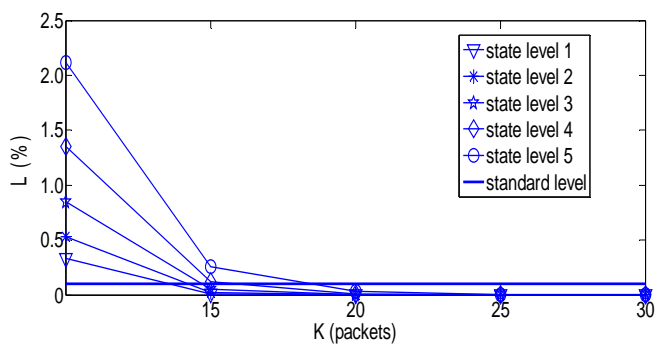

Figure 6 System blocking probability in different channel state levels

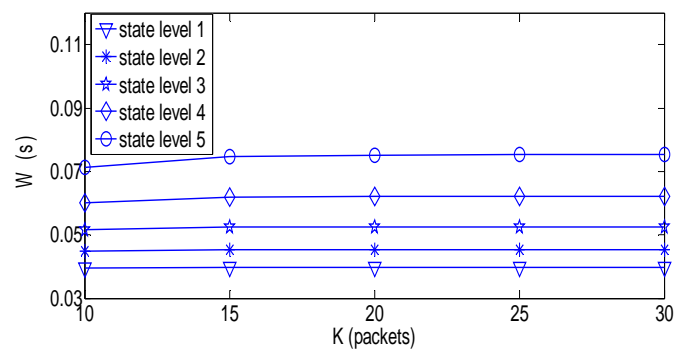

Figure 7 Average sojourn time under different channel state levels

The horizontal line in Figure 6 represents the biggest blocking rate that the system admits (noted as "standard level" in Figure 6). As can be seen from the intersection of various curves and the horizontal line in Figure 6, the worse channel quality is, the bigger buffer size we need to guarantee the system blocking rate reduce to the reference value. Therefore, when channel quality deteriorates, we can increase the buffer size to reduce the system blocking rate. From Figure 7 we can see that the average sojourn time increases as buffer size increases. From previous analysis, we have learned that M2M small data services have delay-tolerance characteristics, which helps to reduce the system blocking rate at the cost of increasing the delay. Comprehensively considering Figure 6 and Figure 7 we can conclude that, when the buffer size increases to a certain value, the blocking rate will reduce to meet the system requirements, moreover, if we continue increasing the buffer size, the blocking probability decreases slowly but the average sojourn time increases continually. Furthermore, it will waste system resources when buffer size is too big. Therefore, we should take the blocking probability and the average sojourn time into account together to get the best system performance.

\section{CONCLUSION}

In this paper, we derived the distribution $G m$ of the arrival process of aggregate traffic of M2M small data services. Then we computed the Hurst parameter $\mathrm{H}$ which is used to measure the self-similarity of M2M aggregate traffic. To simulate the time-varying characteristics of wireless channel, MMPP-2 is used to model the service process of WLAN. By analyzing and solving $G m / M M P P / 1 / K$, we derived the blocking rate and average sojourn time of M2M aggregate traffic under different channel state levels. Considering the delay-tolerance characteristics of M2M small data services, when quality of wireless channel decreases, the blocking rate requirements can be guaranteed by increasing the buffer size reasonably. Our results have some guiding significance for the access network performance analysis and wireless source management of M2M services when the Internet of Things has large-scale application in the worldwide.

\section{ACKNOWLEDGMENT}

This work is supported by National Natural Science Foundation of China (No. 61071074) and Jilin Province Youth Foundation (No.201201031).

\section{REFERENCES}

[1] Gong Qiang, Batta Rajan. A queue-length cutoff model for a preemptive two-priority M/M/1 system. SIAM Journal on Applied Mathematics, v 67, n 1, p 99-115, 2006.

[2] Morris T.D., Perros H.G. Approximate analysis of a discrete-time tandem network of cut-through queues with blocking and bursty traffic. Performance Evaluation, v 17, n 3, p 207-223, May 1993.

[3] Lee Y.D., Van De Liefvoort A., Wallace V.L...Modeling correlated traffic with a generalized IPP. Performance Evaluation, v 40, n 1, p 99-114, March 2000.

[4] Sunggon Kim, Kim Jongwoo, Lee Eui Yong. Stationary distribution of queue length in $\mathrm{G} / \mathrm{M} / 1$ queue with two-stage service policy. Mathematical Methods of Operations Research, v 64, n 3, p 467-480, December 2006.

[5] Jongho Bae, Sunggon Kim. The stationary workload of the G/M/1 queue with impatient customers. Queueing Syst (2010) 64: 253-265 DOI 10.1007/s11134-009-9159-0.

[6] Ching-Chang Kuo, Kuo-Hsiung Wang and W. L. Pearn. The Interrelationship Between N-policy M/G/1/K and F-policy G/M/1/K Queues with Startup Time. Quality Technology \& Quantitative Management Vol. 8, No. 3, pp. 237-251, 2011.

[7] Jau-Chuan Ke, Kuo-Hsiung Wang, Cheng-Hwai Liou. A Single Vacation Model G/M/1/K with N Threshold Policy. The Indian Journal of Statistics 2006, Volume 68, Part 2, pp. 198-226c 2006, Indian Statistical Institute.

[8] Li, Fu, Chi Xue-Fen, Zhang Jia-Sheng. Flow characteristics analysis and modeling of M2M service. CSAE 2012 - Proceedings, 2012 IEEE International Conference on Computer Science and Automation Engineering, v 2, p 459-463, 2012. 